\title{
Lapurdum
}

Euskal ikerketen aldizkaria | Revue d'études basques |

Revista de estudios vascos | Basque studies review

$20 \mid 2017$

Numéro $X X$

\section{Éléments nouveaux sur les attitudes des Bretons envers les étrangers}

\section{Ronan Le Coadic}

\section{OpenEdition}

\section{Journals}

Édition électronique

URL : https://journals.openedition.org/lapurdum/3483

DOI : 10.4000/lapurdum.3483

ISSN : 1965-0655

\section{Éditeur}

IKER

Édition imprimée

Date de publication : 1 janvier 2017

Pagination : 59-71

ISBN : 978-2-95534-135-3

ISSN : $1273-3830$

Référence électronique

Ronan Le Coadic, "Éléments nouveaux sur les attitudes des Bretons envers les étrangers », Lapurdum [En ligne], 20 | 2017, mis en ligne le 01 janvier 2021, consulté le 03 septembre 2021. URL : http:// journals.openedition.org/lapurdum/3483; DOI : https://doi.org/10.4000/lapurdum.3483 


\section{Éléments nouveaux sur les attitudes des Bretons envers les étrangers}

Ronan LE COADIC

Université Européenne de Bretagne

Université Rennes 2, CRBC, ÉA 4451

La presse française se fait régulièrement l'écho d'un risque de « repli identitaire », d'une part, et d'une montée de la xénophobie, d'autre part, deux phénomènes qui sont susceptibles d'être corrélés. Les travaux scientifiques sur le « racisme culturel » considèrent, en effet, qu'au " racisme classique », caractérisé par la hiérarchisation des races et la volonté d'inférioriser les groupes racisés, aurait succédé un " nouveau racisme » (Barker 1981), culturel et différentialiste, qui viserait non pas à inférioriser les victimes du racisme mais à les exclure (Taguieff 1990). La revendication d'un droit à la différence serait donc une façon insidieuse de rejeter l'Autre et de chercher à rester entre soi.

Il convient de préciser, cependant, qu'il serait outrancier de réduire toute forme de mise en avant d'une identité à un « repli » sur soi (cf. Castells 1999: 16-23); on notera, de plus, que les travaux sur le racisme culturel ne font pas l'unanimité : ils sont débattus par la communauté scientifique (cf., notamment, Wieviorka 1998:31-37); enfin, on se gardera de confondre racisme et xénophobie : c'est seulement à cette dernière — c'est-à-dire à l'hostilité 
à l'étranger ${ }^{1}$ - que nous consacrerons nos quelques pages. Ces nuances étant posées, la question de savoir s'il existe une relation entre xénophobie et mise en avant d'une identité particulière reste pertinente. Nous nous poserons cette question à propos des Bretons, réputés très enclins à faire état de leur identité, et appuierons notre étude sur les résultats inédits de deux sondages réalisés en Bretagne historique selon la méthode des quotas : l'un en 2003 auprès d'un échantillon de 1300 personnes (Le Coadic, Ouest-France \& TMO 2003) et l'autre en 2013, auprès de 1003 personnes (BCD \& TMO 2013).

\section{Trop d'étrangers en Bretagne?}

L'un des indicateurs utilisés pour mesurer la xénophobie dans un pays ou une région est fourni par les réponses que ses habitants apportent à la question : " pensez-vous qu'il y a trop d'étrangers dans votre pays (ou votre région) aujourd'hui ?». En France, selon l'institut de sondage BVA, 41 \% de la population considérait en 2003 qu'il y avait « trop d'étrangers en France aujourd'hui » (BVA 2003). Or, seuls 24,6 \% des Bretons répondaient la même année qu'il y avait « trop d'étrangers en Bretagne aujourd'hui » (Le Coadic, Ouest-France \& TMO 2003), soit $40 \%$ de moins que l'estimation fournie par BVA pour la population française.

La différence est encore plus frappante en 2013 : 70 \% de la population française considère qu'il y a « trop d'étrangers en France aujourd'hui » (Ipsos 2013) contre seulement 23,2 \% de la population bretonne qui considère qu'il y a « trop d'étrangers (non français) en Bretagne aujourd'hui » (BCD \& TMO 2013) ${ }^{3}$, soit $66 \%$ de moins que l'estimation fournie par Ipsos pour la population française. Le contraste est éclatant.

On pourra, certes, objecter que si la réponse bretonne est si modérée, c'est que la proportion de personnes d'origine étrangère vivant en Bretagne est nettement inférieure à ce

1. La xénophobie est l'hostilité à ce qui est étranger. Elle est proche de l'ethnocentrisme et du racisme tout en s'en distinguant, nous rappelle Véronique de Rudder : "Parmi les nombreuses notions qui tentent de définir les formes et les contenus des rapports d'hostilité à des groupes définis comme "différents" ou "autres", celle de xénophobie désigne, en toute étymologie, l'hostilité à ce qui est étranger, ou à ce qui en provient. Du point de vue conceptuel, la xénophobie se distingue de l'ethnocentrisme comme du racisme (...). L'ethnocentrisme procède de la valorisation systématique de son propre groupe culturel d'appartenance, pris comme référence, à l'aune de laquelle les autres groupes sont évalués, et du même coup, le plus généralement, jugés inférieurs. Le racisme fonde l'infériorisation, l'hostilité et le refus du "métissage" dans une différence absolutisée par la référence à une nature biologique et héréditaire. Pourtant, la xénophobie partage le plus souvent avec l'ethnocentrisme le système autoréférentiel : la collectivité à laquelle on appartient, ses coutumes, ses valeurs, ses lois, sa religion, sa langue... constituent pour l'un comme pour l'autre la norme du bon, du bien et du vrai. » (Simon \& De Rudder 1990:12)

2. Bretagne à cinq départements, Loire-Atlantique comprise.

3. La précision «non français» a été introduite en 2013 afin d'éviter tout biais concernant le mot «étranger». 
qu'elle est dans l'ensemble de la France. Il est exact, en effet, que peu d'immigrés ${ }^{4}$ vivent en Bretagne: ils ne représentaient que 1,7 \% de la population de la région Bretagne ${ }^{5}$ en 1999, pour une moyenne française de $7,3 \%$, et $2,7 \%$ en 2009 , pour une moyenne française de $8,5 \%$. Leur proportion dans la population bretonne crô̂t néanmoins régulièrement depuis une quarantaine d'années, au point d'avoir quadruplé entre le recensement de 1962 et celui de 1999, alors que sur l'ensemble du territoire français, la part des immigrés est restée relativement stable depuis 1975. De plus, cet argument de la présence étrangère repose sur le présupposé que la xénophobie se développerait à mesure que la proportion d'étrangers croît dans la population alors que les choses sont — heureusement — plus subtiles : « la coïncidence des géographies de la violence, de l'immigration et du vote FN demeure approximative », rappelle en effet Hervé Le Bras. "Elle se vérifie mieux dans les grandes zones urbaines que dans leur périphérie, moins peuplée. On ne peut exclure une influence de la densité de la population ou de la structure du peuplement (Le Bras 1995 : 391).» En outre, à l'échelle locale, les personnes les plus tentées d'affirmer qu'il y a « trop d'étrangers » habitent souvent dans les communes où il y en a le moins (CNCDH 2006).

Donc, la xénophobie ${ }^{6}$ semble être beaucoup moins élevée en Bretagne que dans l'ensemble de la France, ce qui est cohérent avec d'autres indices ${ }^{7}$ et surtout avec l'infériorité chronique des résultats électoraux du Front national en Bretagne par rapport à la moyenne française ${ }^{8}$. Cette faiblesse relative de la xénophobie et du vote Front National en Bretagne ne peut vraisemblablement s'expliquer que par des raisons sociales et culturelles profondes.

4. Rappelons que les populations étrangère et immigrée ne se confondent pas totalement : un immigré n'est pas nécessairement étranger et réciproquement, certains étrangers sont nés en France (essentiellement des mineurs). La qualité d'étranger peut se perdre par acquisition de la nationalité, tandis que la qualité d'immigré est permanente : un individu continue à appartenir à la population immigrée même s'il devient français par acquisition. C'est le pays de naissance, et non la nationalité à la naissance, qui définit l'origine géographique d'un immigré.

5. Région administrative à quatre départements, ne comprenant pas la Loire-Atlantique.

6. Telle, du moins, qu'elle peut être approximativement estimée par la réponse à la question « pensez-vous qu'il y a trop d'étrangers dans votre pays (ou votre région) aujourd'hui ?»

7. Ainsi, par exemple, ce que révélait à propos du mot « étranger » l'étude de sémiométrie menée par la Sofres en 1991 sur les valeurs des Français. Soumis, au sein d'une liste de deux cent dix mots non consensuels et sémantiquement stables, à un échantillon de vingt mille personnes, auxquelles il était demandé d'attribuer à chaque mot une note allant de -3 (très désagréable) à + 3 (très agréable), le mot «étranger » avait été jugé « agréable » par la majorité des Bretons et « désagréable » par la majorité de leurs concitoyens. (Sofres 1991). Renseignements fournis par Yann Battard, Chargé d'études au département de sémiométrie à la Sofres, 11 septembre 1995. Plus récemment, un sondage réalisé en août 2010 sur l'adhésion des Français aux mesures de lutte contre l'insécurité montre que ce sont les habitants de «l'Ouest» (la Bretagne n'est pas clairement isolée) qui sont les moins favorables au « retrait de la nationalité française aux ressortissants d'origine étrangère coupables de polygamie ou d'incitation à l'excision ». (IFOP 2010).

8. Ainsi, aux élections départementales de 2015, le Front National a obtenu 22,23\% des voix en France métropolitaine contre seulement 18,3\% en Bretagne historique. Voir, sur la faiblesse du Front National en Bretagne, les travaux de Jean-Luc Richard (Richard 1999). 
Contrairement à ce qui se produit dans les régions qui accordent une forte proportion de leurs suffrages au Front National, comme le Bassin parisien ou la côte provençale, le tissu social en Bretagne n'est pas en voie de délitement :

(1) « Bassin parisien et littoral provençal ont une structure politique dramatiquement simple : toute atteinte au prestige ou à l'identité nationale se répercute sans intermédiaire au niveau local et familial. Une crise d'identité de la France est ressentie comme une crise d'identité individuelle... Le vote Le Pen doit donc être pris au sérieux. Il indique une dégénérescence des forces politiques intermédiaires qui filtraient jusqu'alors les impulsions immédiates. » (Le Bras 1995 : 216).

Non seulement le tissu social breton n'est pas en voie de délitement mais le lien social est même robuste dans la péninsule. Cela s'explique par un heureux dosage d'ingrédients qui auraient pu, en cas d'excès, s'avérer néfastes : ruralité, bocage ${ }^{9}$, réminiscences catholiques, valeurs cohésives, culture propre, et résistance à l'État centralisateur. Ces facteurs se combinent assez harmonieusement pour faire de la Bretagne une société solidaire, relativement peu en proie à la peur de l'autre (Le Coadic 1998 : 402-418). Cette dernière, néanmoins, n'est pas totalement absente et elle est d'abord corrélée à la préférence politique des personnes interrogées.

\section{La préférence politique}

Sans surprise, ce sont les sympathisants du Front National qui considèrent le plus massivement qu'il y a trop d'étrangers en Bretagne. Ils sont en effet 74,60 \% dans ce cas en 2003 et $47,2 \%$ en 2013.

En revanche, les personnes les moins enclines à dire qu'il y a trop d'étrangers en Bretagne sont, en 2003, celles qui se disent « de gauche » sans plus de précision (10,70 \%), et, à 14,80\%, les sympathisants « régionalistes » (composés à 49,6\% de personnes qui souhaitent l'indépendance de la Bretagne, à 28,8\% de partisans de l'autonomie et à 21,6\% de régionalistes au sens propre du terme).

En 2013, l'étiquette « régionaliste » n'est plus utilisée par l'institut de sondage TMO, qui choisit de mentionner explicitement le parti autonomiste UDB, Union démocratique

9. Selon Hervé Le Bras, « l'augmentation récente de la circulation et de la mobilité locale ont vidé les communautés locales de leurs rôles. Dans les pays de bocage, où le besoin de regroupement était à la source de nombreuses coutumes et règles sociales, la réponse immédiate a été d'amplifier ces règles, mais dans les pays d'agglomération, les coutumes qui visaient au contraire à l'évitement de contacts trop nombreux ont été prises à revers, entrainant la destruction de la communauté locale. Le voisin est devenu un étranger, l'image palpable de cet étranger imaginaire dont on feint de craindre la venue, le miroir d'individus privés de liens sociaux par le changement de leurs rapports immédiats. [...] Tandis qu'à l'ouest et au sud-ouest de la France, les déplacements modernes facilitaient les rencontres entre personnes vivant dans des écarts, c'était l'inverse au Nord-Est, où elles séparaient ceux qui vivaient côte à côte. Paradoxalement, donc, c'est dans les régions de plus grande circulation générale que l'isolement des individus s'est accru. » (Le Bras 1995:408-409) 
bretonne. Or, seules deux catégories de personnes récusent l'idée qu'il y aurait trop d'étrangers en Bretagne : les sympathisants du NPA, Nouveau parti anticapitaliste, d'extrême gauche (0 \%) et ceux de l'UDB ( $0 \%$ également).

Le fait mérite d'être souligné. En effet, selon des représentations répandues en France et souvent relayées par les médias, les revendications autonomistes ou indépendantistes relèveraient d'un repli « tribal » ou « communautariste » qui s'opposerait à l'universalisme et exclurait l'étranger. Or, en examinant les résultats de ces deux sondages, nous constatons, au contraire, que les autonomistes, indépendantistes ou autres régionalistes constituent l'une des sous-populations les moins xénophobes de Bretagne, laquelle est moitié moins xénophobe que la moyenne française.

Cette grande faiblesse de la xénophobie au sein de la mouvance bretonne pourrait paraître surprenante pour deux raisons.

En premier lieu, parce que dans beaucoup d'esprits le nationalisme breton est associé à la collaboration avec l'occupant au cours de la Seconde Guerre mondiale - c'est même devenu un cliché. À ce titre, on pourrait donc s'attendre à de la xénophobie de sa part et même à du racisme. Toutefois, d'une part, il convient, de rappeler que si la collaboration de militants nationalistes bretons pendant la guerre a été une réalité, elle n’a néanmoins concerné qu'une partie de la mouvance bretonne ${ }^{10}$ et, d'autre part et surtout, il est important d'éviter « tout amalgame entre cette époque et le mouvement breton actuel qui, depuis les années 1960, a dans sa grande majorité des fondements et des comportements démocratiques évidents », comme le rappelle l'historien Michel Denis (Denis 2002 : 151).

En second lieu, même sans pratiquer un quelconque amalgame à l'encontre du mouvement breton, on pourrait redouter que sa dimension nationaliste ou paranationaliste débouche sur une forme de racisme. Ainsi que l'écrit Étienne Balibar, en effet, « le racisme sort constamment du nationalisme et, de son côté, le nationalisme sort constamment du racisme » (Balibar 1993 : 80). N'est-il pas indispensable, cependant, pour éviter toute confusion, de préciser ce qu'on entend par « nationalisme » et de distinguer des variantes au sein de cette nébuleuse?

Les définitions du nationalisme abondent et sont parfois fort ingénieuses ${ }^{11}$. Aucune, néanmoins, ne semble parvenir à embrasser l'intégralité du phénomène ni à recueillir l'assentiment de la communauté scientifique en son ensemble. Pourquoi la tâche est-elle si ardue? Selon Benedict Anderson :

(2) « La difficulté vient, en partie de ce qu'on a inconsciemment tendance à hypostasier l'existence du Nationalisme avec un grand N (comme on pourrait le faire de l'Âge avec un grand A) puis à y reconnaître une idéologie. (Si tout le monde a un âge, l'Âge n'est jamais qu'une expression analytique.) La tâche serait plus facile si on considérait qu'il appartenait au

10. Pour une vision d'ensemble de la question, on se reportera avec profit aux actes du colloque international de Brest (Bougeard 2002).

11. Celle d'Ernest Gellner, selon laquelle « le nationalisme est essentiellement un principe politique, qui affirme que l'unité politique et l'unité nationale doivent être congruentes », est l'une des plus connues aujourd'hui (Gellner 1989:11). 
même ordre de phénomène que la "parenté" ou la "religion", plutôt qu'à celui du "libéralisme" ou du "fascisme" (Anderson 2005 : 19). »

À cette remarque judicieuse, on peut ajouter trois compléments. En premier lieu, l'assise sociale du nationalisme est paradoxalement à la fois très étroite et très large. Le nationalisme doctrinal, pensé et théorisé, celui que Michelat et Thomas appellent le « nationalisme des nationalistes » (Michelat \& Thomas 1966 : 14), est généralement cantonné à quelques cercles intellectuels ou militants. En revanche, le nationalisme au sens anthropologique - lié au précédent, qui en est souvent l'expression savante - ou " nationalisme de tout le monde » (Michelat \& Thomas 1966 : 14) est, lui, extrêmement répandu mais fréquemment impensé, voire inconscient. Ainsi que l'écrit Anderson en effet, dans un esprit anthropologique, il faut concevoir le nationalisme " comme une manière d'être-au-monde à laquelle nous sommes tous soumis, plutôt que, simplement, l'idéologie politique de quelqu'un d'autre » (Anderson $2005: 9)$.

En deuxième lieu, chaque nationalisme comporte de multiples facettes. Il est d'usage de distinguer le nationalisme « civique » du nationalisme " ethnique »; toutefois, la réalité est plus complexe : chaque nationalisme mêle composantes affectives, culturelles et idéologiques, et relève à la fois du psychologique et du politique. Se fondant sur l'étude de cinq variables nationalistes ${ }^{12}$ et de huit variables annexes ${ }^{13}$, Michelat et Thomas ont ainsi bâti, en 1962, une typologie ternaire du nationalisme français: « l'échelle souveraineté nationale va représenter le nationalisme idéologique ; l'échelle appartenance au groupe nation et l'indice général de supériorité de la France correspondront à deux formes distinctes du nationalisme affectif (Michelat \& Thomas 1966 : 116). » Ils ont établi de la sorte huit modalités de nationalisme français, qu'ils ont corrélées aux principales familles politiques françaises (gauche, droite et centre). Leur travail pionnier est intellectuellement stimulant même si, par sa date de réalisation (quelques semaines avant la signature des accords d'Évian) comme par la modestie de son échantillon (deux cent vingt-trois étudiants), il présente incontestablement des limites.

Enfin, en troisième lieu, le nationalisme peut, selon les contextes historiques et sociaux, s'inscrire dans des projets de natures diverses. On peut ainsi distinguer, avec Denis Monière, quatre groupes de nationalismes: nationalisme de domination, nationalisme de libération, nationalisme de conservation, et nationalisme de revendication (Monière 2001 : 11-14). Un même nationalisme pourra, selon les époques et les milieux, servir des desseins radicalement différents. Quoi de commun, en effet, entre le nationalisme français des soldats de l'an II, celui des Résistants, celui des défenseurs de l'Algérie française et celui d'ouvriers aujourd'hui victimes de délocalisations?

Si on ne peut, donc, enfermer le phénomène nationaliste dans les petites cases d'un

12. Sentiment d'appartenance au groupe nation, attachement à l'histoire, sentiment que la France est chargée dans le monde d'une mission, représentation du rôle actuel de la France dans le monde et importance accordée à la notion de souveraineté nationale.

13. Attitude à l'égard de la politique coloniale, attitude à l'égard de l'autorité, attitude à l'égard de l'armée, attitude à l'égard des systèmes politico-économiques, attitude à l'égard de la morale et de la religion, attitude à l'égard des races, attitude à l'égard du rôle de la femme, et pessimisme. 
modèle figé, aussi soigné soit-il, parce qu'un même mouvement nationaliste est susceptible, au gré des circonstances, de se glisser d'une case à l'autre, il existe en revanche, à un moment donné de l'histoire et dans une société donnée, des contrastes entre les formes de nationalisme, dont il peut être judicieux de tenir compte; par exemple, entre le nationalisme d'État (qu'il soit de domination ou de conservation, pour reprendre la typologie de Monière) et celui des mouvements minoritaires (nationalismes de contestation ou de libération, selon cette même typologie) qui s'opposent à lui. Il serait intéressant, à cet égard, de comparer les nationalismes breton et français sur la question qui nous occupe, la xénophobie. Malheureusement, si nous avons pu cerner assez précisément la dimension nationaliste bretonne en isolant la catégorie " régionalistes » au sein des préférences politiques exprimées par les sondés, il n'est pas possible de procéder de la même manière en ce qui concerne le nationalisme français car il est présent au sein de différents partis. Nous disposons néanmoins d'un indicateur : le sentiment éprouvé par les sondés lorsqu'ils entendent la Marseillaise, l'hymne national français. Ceci peut nous donner une idée du rapport entre une partie du « nationalisme affectif $»^{14}$ français et la xénophobie.

\section{La Marseillaise}

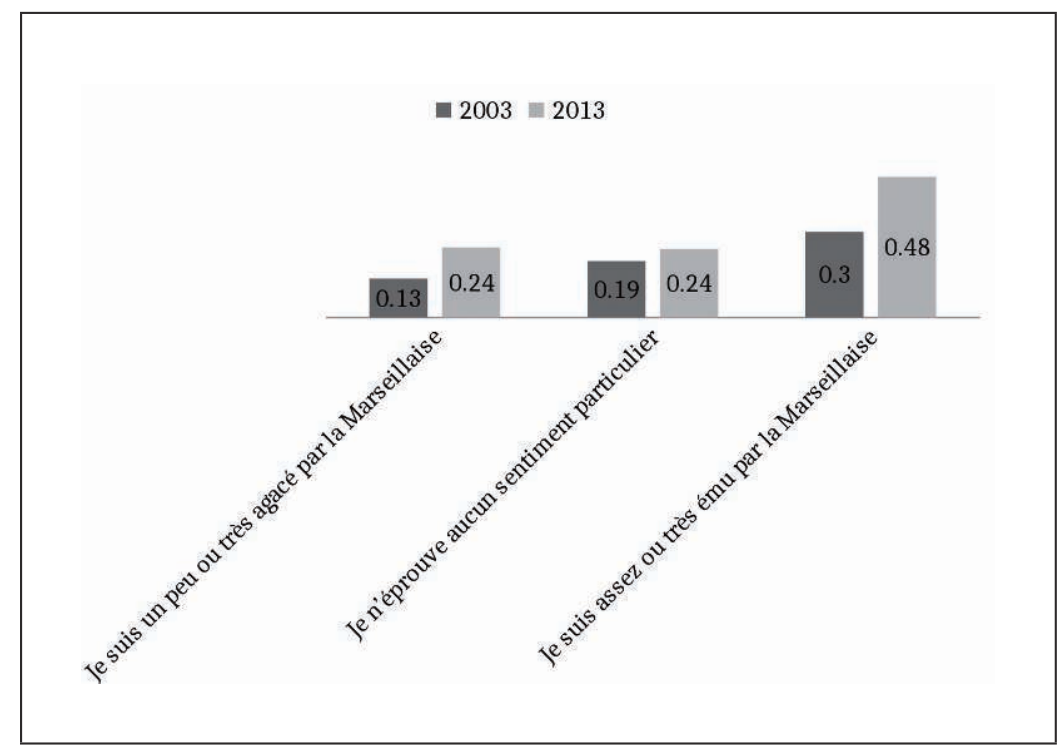

Figure 1 : Proportion de personnes qui déclarent : «Il y a trop d'étrangers en Bretagne aujourd'hui » et sentiment inspiré par la Marseillaise

Le graphique ci-dessus montre, en 2003 comme en 2013, que plus le sentiment éprouvé par les personnes interrogées lorsqu'elles entendent La Marseillaise est positif, plus elles ont tendance à répondre qu'il y a « trop d'étrangers en Bretagne ». Certes, le nationalisme

14. Voir, sur cette question du « nationalisme affectif », Michelat \& Thomas 1966:116. 
français est plus vaste que le simple versant affectif du sentiment d'appartenance au groupe nation tel que l'attachement à l'hymne national le révèle ; il comprend également, comme l'ont montré les politistes Michelat et Thomas, le « sentiment de supériorité de la France » et le "nationalisme idéologique », attaché à la notion de souveraineté nationale (Michelat \& Thomas 1966: 113-124). Il se pourrait néanmoins que nous ayons décelé une relation positive entre l'une des facettes du nationalisme français - le sentiment d'appartenance au groupe national - et la xénophobie, alors que la relation que nous avons trouvée avec le nationalisme breton est, au contraire, négative. De même, en 2003, pour 24,6 \% de Bretons qui pensent, en moyenne, qu'il y a «trop d'étrangers en Bretagne », la proportion tombe à $14,8 \%$ chez les « régionalistes » bretons mais monte à 29,5\% chez les personnes qui se disent « émues» par la Marseillaise. Et en 2013, pour 23,2 \% de Bretons qui pensent, en moyenne, qu'il y a « trop d'étrangers en Bretagne », la proportion tombe à 0,0\% chez les sympathisants de l'Union démocratique bretonne mais monte à $48,4 \%$ chez les personnes qui se disent « émues » par la Marseillaise.

Ces informations, reportées dans le graphique ci-dessous, mériteraient sans doute des investigations ultérieures.

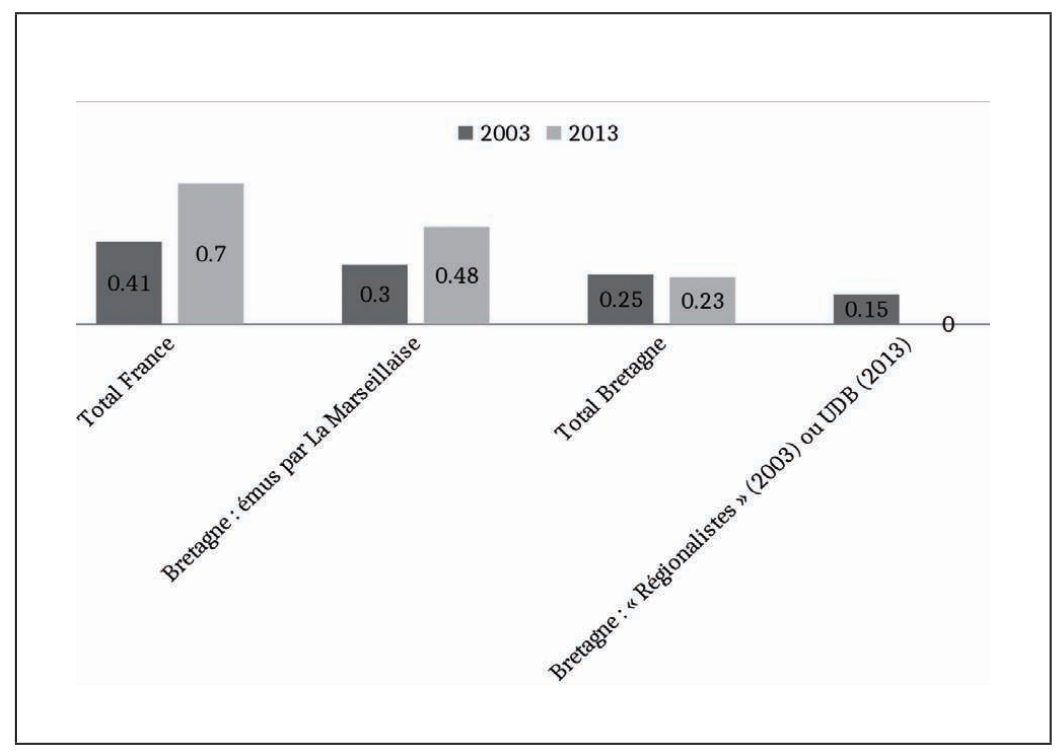

Figure 2: Échelonnement du sentiment selon lequel il y a « trop d'étrangers aujourd'hui »

De tels chiffres doivent être pris avec précaution pour deux raisons. En premier lieu, il est toujours délicat de tirer des conclusions de sondages différents. Nous savons, par l'Ipsos, que 70 \% des Français considèrent en 2013 qu'il y a « trop d'étrangers en France aujourd'hui » et, par TMO, que seuls 23,2 \% des Bretons pensent qu'il y a « trop d'étrangers (non français) en Bretagne » la même année. Rien n'empêche de supposer qu'une proportion importante de Bretons considère qu'il y a « trop d'étrangers » en France mais ce n'est pas le cas en Bretagne... En second lieu, se pose la question de l'inférence causale. Le sondage de TMO fait, en effet, apparaître une relation très significative entre l'âge des personnes interrogées, leur niveau de diplôme, et le sentiment que la Marseillaise leur inspire : les personnes les plus âgées et les moins diplômées (ce qui est lié) sont celles qui sont les plus émues par l'hymne national 
français. Quelle conclusion en tirer ? N'y aurait-il, en réalité, aucun lien entre la Marseillaise et la xénophobie, cette dernière étant exclusivement corrélée à l'âge et au niveau de diplôme? Ou bien les différentes variables évoquées (âge, niveau de diplôme et émotion suscitée par l'hymne national français) concourent-elles ensemble et conjointement aux variations du sentiment qu'il y a « trop d'étrangers » ? Il est difficile de répondre avec certitude. Des entretiens approfondis permettraient sûrement d'en savoir davantage.

Supposons néanmoins que, toutes choses égales par ailleurs, les relations que nous venons de mettre à jour soient confirmées, il conviendrait, pour tenter de les expliquer, de les placer dans une perspective où les attitudes des personnes interrogées seraient considérées comme des ensembles cohérents liés à des tendances profondes : "l'univers est (...) perçu en fonction de schèmes culturels intériorisés et d'expériences vécues dès l'enfance et l'adolescence », expliquent, en effet, Michelat et Simon ; « cet acquis précoce est lui-même plus ou moins réactivé par tout ce qui découle des conditions et du cadre de l'existence adulte » (Michelat \& Simon 1977 : 463).

Or, on sait, en premier lieu, grâce aux travaux d'Annick Percheron sur la socialisation, que le nationalisme français, qui légitime un ordre institutionnel établi, se développe particulièrement tôt chez l'enfant ${ }^{15}$, en lien avec un besoin primordial de sécurisation ; il pourrait — c'est une première hypothèse — être corrélé à une forme d'ethnocentrisme, voire de xénophobie, dans la mesure où, dès l'enfance, « ce qui est différent tend à être jugé non seulement comme autre, mais comme moins bien »(Percheron 1985: 187). En revanche, le nationalisme breton — tel, du moins, qu'il s'exprime depuis les années 1960 —, mû par un sentiment de révolte contre l'État, ne relève pas d'un besoin de sécurisation précoce mais d'une démarche de contestation de l'ordre établi qui se développe plus tardivement dans la vie, généralement à partir de l'adolescence ${ }^{16}$, et qui ne s'accompagne pas du jugement que ce qui est autre est moins bien.

Ceci nous amène à une seconde hypothèse explicative, plus large, qui concerne le rapport à la pluralité culturelle. L'universalisme abstrait des Lumières n'a pas favorisé en France le développement d'une réflexion savante sur la pluralité culturelle, qui aurait pu se propager dans l'ensemble de la société ${ }^{17}$; en outre, l'épopée coloniale s'est effectuée au nom

15. « Dans le cas de la France, on observe très tôt le développement, à côté de l'identité nationale, d'attitudes proprement nationalistes. » (Percheron 1985:188).

16. Morvan Lebesque explique au début de son célèbre ouvrage comment, parfois, "l'âge venu », la « bretonnité » se découvre et se cultive. (Lebesque 1970).

17. Comme l'École de Chicago, premier département universitaire de sociologie des États-Unis, qui plaça la réflexion sur les étrangers dans la ville au cœur de ses travaux. 
de la « mission civilisatrice » de la France ${ }^{18}$; enfin, face à l'immigration, le choix de l'État a été de mener une politique assimilationniste (Noiriel 2005 : 60-69). Tout ceci a pu constituer un terreau favorable à la diffusion de représentations sociales de l'étranger, perçu comme quelqu'un de « mal assimilé », qui vient « profiter de la richesse nationale sans en payer la contrepartie », comme un « concurrent», voire comme un « délinquant» (Wihtol de Wenden 1996 : 155). D’ailleurs, la crainte que la machine assimilationniste se détraque, volontiers mise en avant depuis les années 1980 à propos des populations originaires du Maghreb, ne date pas d'hier, comme le rappelle cette citation de 1912 :

(3) «Il n'est pas certain que la France s'assimile les étrangers. Les conditions ne sont plus les mêmes qu'autrefois. Les étrangers qui viennent en France, Allemands, Italiens notamment, restent groupés : ils ont, beaucoup plus qu'on ne l'avait autrefois, le sentiment de leur nationalité ; ils se fréquentent entre eux, ils gardent leur langue, ils ont des journaux rédigés dans cette langue dans les grandes villes, ils se serrent surtout autour de leurs consuls. Lassimilation de ces éléments hétérogènes est beaucoup plus malaisée qu'autrefois. Elle devient incertaine, notamment dans les pays frontières et dans les grandes villes où les nationaux d'une même origine se concentrent. » (Paul Leroy-Beaulieu, dans l'Économiste français, 1912, cité par Wihtol de Wenden 1996 : 155).

Les Bretons, pris globalement, bénéficient d'une certaine forme de protection contre le racisme, la xénophobie et l'extrémisme qui leur est fournie par le poids particulier de la culture catholique dans la péninsule... Comme le remarque Nonna Mayer, en effet :

(4) « Contrairement à une idée tenace, on constate un lien négatif entre le soutien à l'extrême droite et l'intégration à la communauté catholique et à ses valeurs. À diverses reprises, les évêques de France ont vigoureusement condamné les idées du FN comme contraires au message des Évangiles. » (Mayer 2007 : 434).

Quant aux personnes qui se montrent les plus attachées aux spécificités bretonnes (parmi lesquelles se trouvent celles que l'institut de sondage classe comme "régionalistes » en 2003, puis comme «sympathisants UDB » en 2013), elles sont amenées - par la nature même de la question qu'elles posent à l'État-nation — à réfléchir à la pluralité culturelle ;

18. Cf. Jules Ferry : «Si la Déclaration des droits de l'homme a été écrite pour les Noirs de l'Afrique équatoriale, alors de quels droits allez-vous leur imposer les échanges, les trafics? Ils ne vous appellent pas. [...] Je répète qu'il y a pour les races supérieures un droit, parce qu'il y a un devoir pour elles. Elles ont le devoir de civiliser les races inférieures. » (Débats parlementaires, 28 juillet 1885). Cf., également, Léon Blum : « Nous avons trop l'amour de notre pays pour désavouer l'expansion de la pensée et de la civilisation françaises. Nous admettons le droit et même le devoir des races supérieures d'attirer à elles celles qui ne sont pas parvenues au même degré de culture. » (Le Populaire, 17 juillet 1925). Voir, plus globalement, Le Cour Grandmaison 2009. 
en outre, elles se comparent volontiers aux peuples que la France a colonisés ${ }^{19}$; enfin, elles établissent souvent un parallèle entre le sort de leurs parents ou grands-parents exilés à Paris et celui des immigrés d'hier ou d'aujourd'hui ${ }^{20}$. Peut-être tout cela peut-il contribuer à expliquer la faible xénophobie que nous avons constatée dans ce milieu?

En conclusion, le présent article fait apparaître trois résultats majeurs : en premier lieu, l'hostilité à la présence étrangère semble moins élevée en Bretagne que dans l'ensemble de la France; en second lieu, elle est plus faible chez les sympathisants régionalistes, autonomistes ou indépendantistes que dans le reste de la population bretonne; enfin, elle est environ deux fois plus élevée chez les personnes émues par la Marseillaise que dans le reste de la population bretonne.

Nous avons interprété la faible xénophobie bretonne comme une conséquence du lien social et des valeurs cohésives de la société bretonne. Quant aux deux autres résultats, nous les avons interprétés en termes de socialisation, d'une part (le nationalisme français se développe précocement chez l'enfant, tandis que le nationalisme breton apparaît plutôt à l'adolescence) et en termes de modèle culturel, d'autre part (l'universalisme abstrait n'incite guère à réfléchir à la pluralité culturelle, tandis que le sentiment d'appartenir à un groupe spécifique, voire à une minorité laminée, favorise ce type de réflexion).

Ces résultats - en particulier les deux derniers — sont totalement nouveaux. Ils ont d'abord une portée locale, relative à l'étude de la société bretonne ; mais, s'ils sont confirmés à l'avenir, ils pourront s'inscrire dans une réflexion plus vaste sur les différentes logiques nationalistes.

19. Le parti autonomiste UDB, Union démocratique bretonne, a été créé à la suite de la guerre d'Algérie par des militants qui établissaient un parallèle entre la situation algérienne et la Bretagne ; ce parti a d'ailleurs longtemps diffusé une brochure intitulée "Bretagne = colonie ». Le chanteur breton Youenn Gwernig avait dans son répertoire plusieurs titres sur ce thème, dont « les derniers bougnoules». Enfin, une grande partie de la littérature bretonne des années 1970 traitait de ce parallèle, notamment les textes de Xavier Grall. Voir, sur ce point, les travaux de Nathalie Caradec (Cf. Caradec 2013, notamment).

20. Le principal ouvrage scientifique relatif aux migrations bretonnes des années 1970 établit, précisément, un parallèle entre les Bretons et les immigrés d'origine étrangère : « De nombreux migrants bretons ont conscience de faire partie d'une vague migratoire à caractère régional, national, ethnique; ils ont été précédés selon les professions par des Européens - Italiens, Espagnols, Portugais - ils se sont retrouvés dans la même vague que les Africains blancs Algériens, Tunisiens - enfin se voient suivis par les Africains noirs et parfois des Orientaux, comme les Turcs. "Les Bretons maintenant dans le bâtiment, dit un migrant, c'est comme les Italiens après la guerre, prêts à travailler très dur avant de repartir chez eux les poches pleines ". De même que la guerre d'Algérie, qui a pu susciter une réflexion renouvelée sur les identités régionales en métropole, cette situation des Bretons à Paris a joué un rôle peut-être non négligeable pour eux comme élément de différenciation et d'auto-identification, de même que l'image de soi renvoyée par l'autre, du type de celle rapportée par un immigré breton manœuvre sur un chantier à qui un immigré kabyle demande si, faisant un tel travail, il n'est pas plus proche des Kabyles que des Français. » (Prado \& Barbichon 1978:94-95) 
Il convient, à présent, de vérifier nos résultats sur des échantillons plus vastes et, surtout, de mener des études qualitatives, en particulier des entretiens approfondis, qui pourront permettre de faire émerger les univers symboliques sous-jacents aux attitudes constatées.

\section{Bibliographie}

Anderson, Benedict. 2005. L’imaginaire national. Paris : La Découverte.

Balibar, Etienne. 1993. Racisme et nationalisme : une logique de l'excès. In Michel Wieviorka (ed.), Racisme et modernité. Paris : Éditions La Découverte.

Barker, Martin. 1981. The new racism : conservatives and the ideology of the tribe. London: Junction Books.

BCD \& TMO. 2013. Baromètre Bretagne culture diversité. http://bcdiv.org/les-resultats-dusondage-bcd-en-ligne/ (18 August, 2014).

Bougeard, Christian (ed.). 2002. Bretagne et identités régionales pendant la Seconde Guerre mondiale. Brest : CRBC.

BVA. 2003. Xénophobie, antisémitisme, racisme et antiracisme en France. 1-58.

Caradec, Nathalie. 2013. Résonances... Des écrivains bretons à l'écoute des intellectuels et écrivains de la négritude et de la décolonisation. In Ronan Le Coadic (ed.), De la domination à la reconnaissance: Antilles, Afrique et Bretagne, 263-281. Rennes: Presses Universitaires de Rennes (PUR).

Castells, Manuel. 1999. L'Ere de l'information, tome II : Le Pouvoir de l'identité. Paris : Fayard.

CNCDH. 2006. Rapport de la Commission nationale consultative des droits de l'homme présenté à Monsieur le premier ministre. http://lesrapports.ladocumentationfrancaise. fr/BRP/074000226/0000.pdf.

Cour Grandmaison, Olivier Le. 2009. La République impériale : politique et racisme d’État. Paris : Fayard.

Denis, Michel. 2002. Le mouvement breton pendant la guerre. Un bilan. In Christian Bougeard (ed.), Bretagne et identités régionales pendant la Seconde Guerre mondiale. Brest : CRBC.

Gellner, Ernest. 1989. Nations et nationalisme. Paris : Payot.

IFOP. 2010. Sondage sur l'adhésion aux mesures de lutte contre l'insécurité. 1-13.

Ipsos. 2013. Les nouvelles fractures. http://www.ipsos.fr/ipsos-public-affairs/actualites/201301-24-france-2013-nouvelles-fractures (29 May, 2015).

Le Bras, Hervé. 1995. Les trois France. Paris : Éditions Odile Jacob.

Le Coadic, Ronan, Ouest-France \& TMO. 2003. Enquête sur l'identité bretonne. Rennes: TMO et Ouest-France.

Le Coadic, Ronan. 1998. Lidentité bretonne. Rennes: Presses Universitaires de Rennes (PUR) et Terre de Brume.

Lebesque, Morvan. 1970. Comment peut-on être breton? Essai sur la démocratie française. Paris: Seuil.

Mayer, Nonna. 2007. Comment Nicolas Sarkozy a rétréci l'électorat Le Pen. Revue française de science politique 57 (3). 429. doi : 10.3917/rfsp.573.0429 (4 August, 2010).

Michelat, Guy \& Michel Simon. 1977. Classe, religion et comportement politique. Paris : Presses 
de la Fondation nationale des sciences politiques.

Michelat, Guy \& Jean-Pierre H. Thomas. 1966. Dimensions du nationalisme. Armand Colin.

Monière, Denis. 2001. Pour comprendre le nationalisme au Québec et ailleurs. Montréal : Presses de l'Université de Montréal.

Noiriel, Gérard. 2005. Etat, nation et immigration : Vers une histoire du pouvoir. Editions Gallimard.

Percheron, Annick. 1985. La socialisation politique : défense et illustration. In Madeleine Grawitz \& Jean Leca (eds.), Traité de science politique, vol. 3, 165-236. Paris : PUF.

Prado, Patrick \& Guy Barbichon. 1978. Vivre sa ville : migrants bretons et champ urbain. Paris : Centre d'ethnologie française, CNRS-DGRST.

Richard, Jean-Luc. 1999. Le Front National en Bretagne occidentale. Paris : Cevipof.

Simon, Pierre-Jean \& Véronique De Rudder. 1990. Questions de vocabulaire : ethnocentrisme, xénophobie. Migrants-Formation (80).

Sofres. 1991. Valeurs des Français, étude réalisée par le Département de sémiométrie.

Taguieff, Pierre-André. 1990. La Force du préjugé : essai sur le racisme et ses doubles. (Tel 162). Paris : Gallimard.

Wieviorka. 1998. Racisme : une introduction. La Découverte.

Wihtol de Wenden, Catherine. 1996. L'Autre au quotidien. In Bertrand Badie \& Marc Sadoun (eds.), L’Autre. Études réunies pour Alfred Grosser, 151-165. Paris: Presses de Sciences Po. 